\title{
A STRONG MAXIMUM MODULUS THEOREM FOR MAXIMAL FUNCTION ALGEBRAS
}

\author{
BY
}

H. S. BEAR

1. Introduction. In [1] and [3] it is shown that if $A$ is a maximal closed subalgebra of the algebra $C(X)$ of all continuous complex-valued functions on the compact Hausdorff space $X$, then the Silov boundary of $A$ is all of $X$. The following is an equivalent statement: if $A$ is a maximal closed subalgebra of $C(X)$ and $A$ is considered as an algebra of functions on its maximal ideal space $\Sigma(A)$, with $X$ considered as a subset of $\Sigma(A)$, then the Silov boundary of $A$ in $\Sigma(A)$ is $X$. The canonical example of a maximal algebra is the algebra of all continuous functions on $C=\{z:|z|=1\}$ which have continuous extensions to $K=\{z:|z| \leqq 1\}$ which are analytic on the interior of $K$ (see [8]).

For this example, the stronger statement holds that the maximum modulus of a nonconstant function is assumed only on the Silov boundary. We show in $\$ 4$ that this strong maximum modulus property holds for all essential (see 2 for definitions) maximal algebras.

In $\$ 3$ we continue the study of the essential set of an algebra started in [1]. The relationship between the maximal ideal spaces of an algebra and its restriction to its essential set is made explicit. This result makes clear the extent to which the maximum modulus theorem of $\S 4$ applies. This section is based on Theorem 1, which is due to R. S. Pierce.

2. Definitions. We will use the following notation and nomenclature throughout.

$X$ is an arbitrary compact Hausdorff space. $C(X)$ is the algebra of all continuous complex-valued functions on $X$, with the algebraic operations carried out pointwise, and the norm $\|f\|=\sup \{|f(x)|: x \in X\}$.

" $A$ is an algebra on $X$ " will mean that $A$ is a proper closed subalgebra of $C(X)$, containing the constants, and separating points of $X$. " $A$ is a maximal algebra on $X$ " will mean that $A$ is an algebra on $X$ and $A$ is contained in no other proper closed subalgebra of $C(X)$.

The maximal ideal space of $A$ with the Gelfand topology will be denoted $\Sigma(A)$. Equivalently, $\Sigma(A)$ is the space of all nontrivial continuous complex homomorphisms of $A$ with the weak* topology. If $A$ is an algebra on $X$, then $\Sigma(A)$ is a compact Hausdorff space, and $X$ is homeomorphic to a subset of $\Sigma(A)$. We shall always assume, therefore, that $X \subset \Sigma(A)$, and that $A$ can be considered as an algebra either on $X$ or on $\Sigma(A)$.

Presented to the Society, April 19, 1958 under the title $A$ strong maximum modulus property for maximal function algebras; received by the editors May 6, 1958. 
The Silov boundary of an algebra $A$ on $X$ is that unique minimal closed subset of $X$ on which each function in $A$ attains its maximum modulus. If $A$ is an algebra on $X$, then the Silov boundary of $A$ in $\Sigma(A)$ is a subset of $X$.

If $A$ is an algebra on $X$ and $E \subset X$ and $f \in A$, then $f \mid E$ is the restriction to $E$ of the function $f$, and $A \mid E$ is the algebra of all such restrictions.

The essential set [1] of $A$ in $X$ is that unique minimal closed subset $E$ of $X$ such that if $f$ is any continuous function on $X$ and $f|E \in A| E$, then $f \in A$. That is, the essential set is the hull of the largest closed ideal of $C(X)$ which is contained in $A$. We will say that $A$ is an essential algebra on $X$ if the essential set for $A$ is all of $X$.

3. Relationship between $\Sigma(A)$ and $\Sigma(A \mid E)$. In [1] it is shown that if $A$ is an algebra on $X$ and $E$ is the essential set of $A$ in $X$, then $\Sigma(A)=X$ if and only if $\Sigma(A \mid E)=E$. That is, cutting an algebra down to the essential set does not destroy the fact that the algebra is defined on its maximal ideal space. There remains the question of what happens to the maximal ideal space of a nonessential algebra if the algebra is restricted to its essential set. This is answered in Corollary 2 below.

Theorem 1 (R. S. Pierce). If $A$ is an algebra on $X$ with essential set $E$ in $X$, then every $f \in A$ which is zero on $E$ is zero on $\Sigma(A) \sim(X \sim E)$.

Proof. We shall prove the following equivalent assertion: if $h$ is a homomorphism on $A$ and $h(f) \neq 0$ for some $f$ which is zero on $E$, then $h$ is evaluation at a point of $X \sim E$. Assume that $f \in A, f=0$ on $E$, and $h(f)=\alpha \neq 0$. We show that $h$ extends to a homomorphism $\tilde{h}$ of $C(X)$. Let $\tilde{h}(g)=\alpha^{-1} h(f g)$ for any $g \in C(X) ; h(f g)$ is defined, since $f$ is zero on $E$, and hence $f g \in A$. If $g \in A$, $\tilde{h}(g)=\alpha^{-1} h(f g)=\alpha^{-1} h(f) h(g)=h(g)$, so $\tilde{h}$ is an extension of $h$. It is clear that $\tilde{h}$ is linear, and we check only that $\tilde{h}$ is continuous and multiplicative. For $g_{1}, g_{2} \in C(X)$,

$$
\begin{aligned}
\tilde{h}\left(g_{1} g_{2}\right) & =\alpha^{-1} h\left(f g_{1} g_{2}\right)=h(f) \alpha^{-2} h\left(f g_{1} g_{2}\right)=\alpha^{-2} h\left(f g_{1} f g_{2}\right) \\
& =\alpha^{-1} h\left(f g_{1}\right) \alpha^{-1} h\left(f g_{2}\right)=\tilde{h}\left(g_{1}\right) \tilde{h}\left(g_{2}\right),
\end{aligned}
$$

so $\tilde{h}$ is multiplicative. For any $g \in C(X)$,

$$
|\tilde{h}(g)|=(1 /|\alpha|)|h(f g)| \leqq(1 /|\alpha|)\|h\|\|f g\| \leqq(\|f\| /|\alpha|)\|g\| ;
$$

so $\tilde{h}$ is continuous. There is, therefore, a point $x \in X$ such that $\tilde{h}(g)=g(x)$ for all $g \in C(X)$. Since $0 \neq \alpha=h(f)=\tilde{h}(f)=f(x)$, and $f$ is zero on $E, x \in E$.

Corollary 1. If $A$ is an algebra on $X$ with essential set $E$ in $X$ and $E \neq X$, then $X \sim E$ is open in $\Sigma(A)$.

Proof. Let $x_{0} \in X \sim E$ and $f$ be a continuous real valued function which is zero on $\Sigma(A) \sim(X \sim E)$ and not zero at $x_{0}$. This $f$ is in $A$ by the definition of $E$ as the essential set in $X$, and Theorem 1. The set $\{x: f(x) \neq 0\}$ is open in $\Sigma(A)$, contains $x_{0}$, and is contained in $X \sim E$. 
Corollary 2. If $A$ is an algebra on $X$ with essential set $E$ in $X$ and $E \neq X$, then $\Sigma(A \mid E)=\Sigma(A) \sim(X \sim E)$.

Proof. Let $\Sigma(A) \sim(X \sim E)=F$. By Theorem 1, if $f, g \in A$ and $f|E=g| E$, then $f|F=g| F$. For any $f \in A \mid E$, let $\tilde{f}$ be the unique function on $F$ such that $\tilde{f} \mid E=f$ and $\tilde{f} \in A \mid F$. Each point $x$ of $F$ gives rise to a distinct homomorphism $h_{x}$ of $A \mid E: h_{x}(f)=\tilde{f}(x)$. We show next that every homomorphism of $A \mid E$ is of this form. Let $h$ be a homomorphism of $A \mid E$ and note that $h$ can be extended to a homomorphism $\tilde{h}$ of $A$ by $\tilde{h}(f)=h(f \mid E)$, for each $f \in A$. There is a point $x \in \Sigma(A)$ such that $h(f)=f(x)$ for all $f \in A$. To see that $x \in X \sim E$, consider a function $f \in A$ which is zero on $E$ and one at $x$; for this $f, 1=f(x)=\tilde{h}(f)$ $=h(f \mid E)=h(0)=0$. Therefore, each homomorphism of $A \mid E$ can be represented as evaluation (of $\bar{f}$ ) at a point of $F$.

Corollary 3. If $A$ is an algebra on $X$, then $A$ is essential on $X$ if and only if $A$ is essential on $\Sigma(A)$. If $E$ is the essential set of $A$ in $X$, then $\Sigma(A) \sim(X \sim E)$ is the essential set of $A$ in $\Sigma(A)$.

Proof. Let $E$ and $F$ denote the essential sets of $A$ in $X$ and $\Sigma(A)$ respectively. Since the complement of the essential set $F$ is contained in the Silov boundary, we have $\Sigma(A) \sim F \subset X$, or $F \supset \Sigma(A) \sim X$. By the definition of $E$, we have further that $F \supset \Sigma(A) \sim(X \sim E)$. Therefore, if $E=X, F=\Sigma(A)$. On the other hand, if $A$ is not essential on $X$, then $X \sim E$ is open in $\Sigma(A)$ and by Theorem 1 every continuous function zero on $\Sigma(A) \sim(X \sim E)$ is in $A$. Hence $A$ is not essential on $\Sigma(A)$, and $F \subset \Sigma(A) \sim(X \sim E)$. We have already noted the other inclusion, so $F=\Sigma(A) \sim(X \sim E)$.

4. Maximal algebras. We proceed to our main theorem via the following two lemmas.

Lemma 1. If $A$ is an essential maximal algebra on $X$, and $f=u+i v \in A$, and $u$ is constant on $X$, then $f$ is constant on $\Sigma(A)$.

Proof. If $u(x)=c$ for all $x \in X$, then the function $(-i)(f-c)$ is in $A$ and equals $v$ on $X$. But Helson and Quigley [4] and the author [1] have shown that an essential maximal algebra contains no nonconstant real functions. Therefore, $v$ is constant on $X$, and hence $f$ is constant on $\Sigma(A)$.

Lemma 2. If $A$ is an essential maximal algebra on $X$ and $x_{0} \in \Sigma(A) \sim X$, then there is a positive Borel measure $\mu$ whose support is all of $X$ such that $f\left(x_{0}\right)$ $=\int_{X} f d \mu$ for all $f \in A$.

Proof. Let $x_{0} \in \Sigma(A) \sim X$, and $\pi(f)=f\left(x_{0}\right)$ for all $f \in A ; \pi$ is a linear functional of norm one on $A \mid X$, and can be extended to a linear functional of norm one on all of $C(X)$. Such a functional can be represented as an integral $\pi(f)=\int_{X} f d \mu$ for some complex Borel measure $\mu$ of total variation one. Since the function identically one is in $A$, we have $1=\pi(1)=\mu(X)$, and it follows 
that $\mu$ is actually a real positive measure. Now suppose that $\mu(U)=0$ for some open subset $U$ of $X$, and let $E=X \sim U$. It is shown in [1] that if $A$ is a maximal essential algebra on $X$, then $A \mid E$ is uniformly dense in $C(E)$ for any proper closed subset $E$ of $X$. Since $\mu$ is carried on $E, \pi$ is actually a continuous functional on $C(E)$ which is multiplicative on the dense subalgebra $A \mid E$. It follows that $\pi$ is a homomorphism of $C(E)$, and can be represented as evaluation at a point of $E$. Thus $\pi(f)=f\left(x_{1}\right), x_{1} \in E$, for all $f \in C(E)$ and in particular all $f \in A$. That is, $f\left(x_{0}\right)=f\left(x_{1}\right)$ for all $f \in A$, which contradicts the fact that $A$ must separate points of $\Sigma(A)$.

Theorem 2. If $A$ is an essential maximal algebra on $X$, and $\left|f\left(x_{0}\right)\right|=\|f\|$ for some $f \in A$ and some $x_{0} \in \mathcal{\Sigma}(A) \sim X$, then $f$ is constant.

Proof. Suppose $f \in A$ and $\left|f\left(x_{0}\right)\right|=\|f\|$ and $x_{0} \in \Sigma(A) \sim X$. Let $f\left(x_{0}\right)$ $=\|f\| e^{i \alpha}, \alpha$ real, and consider the function $g=u+i v=e^{-i \alpha} f$. Then $g \in A$, $\|g\|=g\left(x_{0}\right)=u\left(x_{0}\right)$, and $u(x) \leqq u\left(x_{0}\right)$ for all $x \in \Sigma(A)$. Let $\mu$ be a positive measure on $X$ representing evaluation at $x_{0}: h\left(x_{0}\right)=\int_{X} h d \mu$ for all $h \in A$. We have $g\left(x_{0}\right)=u\left(x_{0}\right)=\int_{X} u d \mu+i \int_{X} v d \mu=\int_{X} u d \mu$. Unless $u$ is constant on $X, \int_{X} u d \mu$ $<u\left(x_{0}\right)$, since the support of $\mu$ is all of $X$ and $u(x) \leqq u\left(x_{0}\right)$. Hence $u$ is constant on $X$, and therefore by Lemma $1, g$ is constant on $\Sigma(A)$. It follows that $f$ is constant.

The two lemmas and Theorem 2 have all had the hypothesis that $A$ is an essential algebra on $X$. It is clear that Theorem 2 is false without this assumption, for in a nonessential algebra each constant has arbitrary continuous extensions off the essential set. We show below what can be said about a nonessential algebra by using Theorem 1 in connection with Theorem 2 .

Theorem 3. If $A$ is a maximal algebra on $X$ with essential set $E$ in $X$, $E \neq X$, then any function which assumes its maximum modulus at a point of $\Sigma(A) \sim X$ is constant on $\Sigma(A) \sim(X \sim E)$.

Proof. $A \mid E$ is an essential maximal algebra on $E$, and $\Sigma(A \mid E)=\Sigma(A)$ $\sim(X \sim E)$. Theorem 2 then applies.

5. Remarks and questions. It is obvious that Theorem 2 has no content if $A$ is maximal on $X$ and $\Sigma(A)=X$. None of the maximal algebras known to the author have this property (e.g., Bishop [2]; Rudin [6]; Wermer [9]). It is a natural question whether a maximal algebra is necessarily maximal only on a part of its maximal ideal space.

Rudin has studied algebras defined on the unit disc in the complex plane with the property that the Silov boundary is contained in the unit circle $[5 ; 7]$. The question here is whether this maximum modulus property for algebras characterizes subalgebras of the analytic functions, and in [7] the answer is shown to be no. The question remains open, however, if we insist that the maximal ideal space be the disc. One can replace the maximum 
modulus assumption by the assumption that the algebra be maximal on the circle, which implies the maximum modulus property, and ask whether this, with the necessary (see [9]) assumption that the maximal ideal space is the disc, characterizes the algebra of all analytic functions continuous on the disc.

A final question related to the preceding two is the following: Suppose that $A$ is an antisymmetric algebra ( $A$ contains no nonconstant real functions) and the Silov boundary of $A$ in $\Sigma(A)$ is $X \neq \Sigma(A)$. Then [1] $A$ is contained in an algebra $A_{1}$ which is maximal with respect to the properties: (i) $\Sigma\left(A_{1}\right)$ $=\Sigma(A)$, and (ii) the Silov boundary of $A_{1}$ is $X$. Is $A_{1} \mid X$ maximal? If so, then the maximum modulus property plus antisymmetry is roughly the same as maximality on the boundary. Also, our Theorem 2 would hold for arbitrary (not necessarily maximal) antisymmetric algebras. Note that antisymmetry is a necessary assumption here; i.e., maximal algebras are antisymmetric [4].

\section{REFERENCES}

1. H. S. Bear, Complex function algebras, Trans. Amer. Math. Soc. vol. 90 (1959) pp. 383393.

2. Errett Bishop, On the structure of certain measures, Duke Math. J. vol. 25 (1958) pp. 283-290.

3. Paul Civin, A maximum modulus property of maximal subalgebras, Proc. Amer. Math. Soc. vol. 10 (1959) pp. 51-54.

4. Henry Helson and Frank Quigley, Maximal algebras of continuous functions, Proc. Amer. Math. Soc. vol. 8 (1957) pp. 111-114.

5. Walter Rudin, Analyticity and the maximum modulus principle, Duke Math. J. vol. 20 (1953) pp. 449-458.

6. - Subalgebras of spaces of continuous functions, Proc. Amer. Math. Soc. vol. 7 (1956) pp. 825-830.

7. - On the structure of maximum modulus algebras, Notices Amer. Math. Soc., Abstract 543-20, June, 1958.

8. John Wermer, On algebras of continuous functions, Proc. Amer. Math. Soc vol. 4 (1953) pp. 866-869.

9. - Subalgebras of the algebra of all complex-valued continuous functions on the circle, Amer. J. Math. vol. 78 (1956) pp. 225-242.

UNIVERSITY OF WASHINGTON,

Seattle, Washinciton 